\title{
Rotating Magnets Produce a Prompt Analgesia Effect in Rats
}

Zhong Chen

Southern Medical University (China)

Hui Ye

Loyola University Chicago, hye1@luc.edu

Haiyun Xu

Shantou University

Shukang An

Southern Medical University (China)

Anmin Jin

Southern Medical University (China)

See next page for additional authors

Follow this and additional works at: https://ecommons.luc.edu/biology_facpubs

Part of the Biology Commons

\section{Recommended Citation}

Z. Chen, H. Ye, H. Xu, S. An, A. Jin, C. Zhou, and S. Yang, "Rotating magnets produce a prompt analgesia effect in rats," Progress In Electromagnetics Research M, Vol. 27, 203-217, 2012.

This Article is brought to you for free and open access by the Faculty Publications and Other Works by Department at Loyola eCommons. It has been accepted for inclusion in Biology: Faculty Publications and Other Works by an authorized administrator of Loyola eCommons. For more information, please contact ecommons@luc.edu.

\section{(c) $(\mathrm{i})($}

This work is licensed under a Creative Commons Attribution-Noncommercial-No Derivative Works 3.0 License.

(c) The Electromagnetics Academy, 2012. 


\section{Authors}

Zhong Chen, Hui Ye, Haiyun Xu, Shukang An, Anmin Jin, Chusong Zhou, and Shaoan Yang 


\title{
ROTATING MAGNETS PRODUCE A PROMPT ANALGESIA EFFECT IN RATS
}

\section{Zhong Chen ${ }^{1, *}$, Hui $\mathrm{Ye}^{2}$, Haiyun $\mathrm{Xu}^{3}$, Shukang $\mathrm{An}^{1}$, Anmin Jin ${ }^{1}$, Chusong Zhou ${ }^{1}$, and Shaoan Yang ${ }^{1}$}

${ }^{1}$ Department of Spinal Surgery, Southern Medical University, Zhujiang Hospital, GuangZhou 510282, China

${ }^{2}$ Department of Biology, Loyola University, Chicago, IL 60660, US

${ }^{3}$ Department of Anatomy, Medical College, Shantou University, Guangdong 515041, China

\begin{abstract}
The beneficial effects of chronic/repeated magnetic stimulation on humans have been examined in previous studies. Although pain relief effects have been reported several weeks after magnetic treatment, no report is available regarding the prompt effect of magnetic stimulations. In this study, a novel apparatus was developed to generate time-varying magnetic fields with rotating magnets. Adult, conscious rats were exposed to the rotating magnets in a posture in which their spines were parallel to the induced electric current. The magnetic field suppressed the paw withdrawal reflex in the anesthetized rats, and the suppression effect disappeared 5 minutes after magnets stopped rotating. The tail flick (TF) latency and mechanic withdrawal thresholds (MWT) of the rats were significantly increased by the rotating magnets; the increases positively correlated with the velocity and period of the magnet rotating. These analgesia effects recovered to the baseline level 30 minutes after magnets stopped rotating. A biophysics model was proposed to qualitatively understand the mechanism of pain inhibition by the rotating field. The prompt analgesia effect of the rotating magnets and its rapid recovery encourage the application of this technique as a promising new analgesia and anesthesia method.
\end{abstract}

Received 9 October 2012, Accepted 21 November 2012, Scheduled 27 November 2012

* Corresponding author: Zhong Chen (chenzhong@smu.edu.cn). 


\section{INTRODUCTION}

Electric-magnetic stimulations have been used as an alternative method when pharmacological therapy titrated to maximal doses failed to provide an appropriate analgesia effect. In applying this technique for pain relief, electrodes are implanted into the spinal canal so that spinal cord stimulation (SCS) can be performed repeatedly for the treatment of intractable pain. However, its pervasion was impeded by some complications occurred in $11 \%-45 \%$ of the treated patients and by the incurred great expense [1-5]. Currently, studies are using static magnetic fields as a replacement of SCS $[6,7]$ and pain relief has been observed in certain subsets of patients with chronic, nonmalignant pain, probably via its effects on inflammation [8]. In a recent metaanalysis based on 11 strictly controlled clinical studies the slow and mild analgesia effects of the static magnetic fields were verified [9], although the same methods failed to provide better therapeutic effects in some other studies [10,11]. Generally, pain relief by static magnetic fields occurred several weeks after treatment $[12,13]$, suggesting that the electric pulses conducted along the axonal membrane were not promptly blocked by the magnetic field. Any static magnetic fields with the intensity lower than 5 Tesla $(\mathrm{T})$ are believed to be unable to block the electric conduction in nerve fibers [14]. Indeed, an early study [15] indicated that the intensity of magnetic field must be more than $24 \mathrm{~T}$ to affect the axonal conduction, which posed technique challenges for the clinical application of static magnetic fields.

Time-varying magnetic fields have been used to overcome this difficulty, by inducing an electric current which may block axonal conduction. Recent studies have tested effects of repetitive transcranial magnetic stimulation (rTMS). For example, the rTMS toward the motor cortex and dorsolateral prefrontal cortex was reported to suppress chronic central pain and show slow and limited analgesia effects. Unfortunately, this stimulation in the brain area induced certain complications such as nausea, headache, seizure, and so on $[16,17]$. Alternatively, researchers have tested the pulsed electromagnetic fields for pain relief in patients with osteoarthritis [18], rheumatoid arthritis, or fibromyalgia [19]. However, it has not been known if a rotating magnetic field (RMF) has any prompt pain relief effect in animals and humans. In this study, a novel apparatus was designed to generate RMF and the field was applied to the lumbar region of the anaesthetized and conscious rats. The RMF was able to produce a prompt analgesia effect in rats, which recovered to normal in a relatively short period of 30 minutes. These results encourage exploring the application of RMF as a promising new analgesia and 
anesthesia method.

\section{MATERIAL AND METHODS}

Experiments were performed on adult male and female SpragueDawley rats (provided by the animal center of Southern Medical University, Guang Zhou, China), which were housed in single cages under a 12-h alternating light/dark cycle with free accesses to food and water. We kept the animals in a comfort environment (temperature: $23 \pm 2^{\circ} \mathrm{C}$; relative humidity: $55 \pm 10 \%$ ). To minimize potential animal stress associated with environment changes, we performed the experiments in the same room and at approximately the same time of the day $(8 \mathrm{Am}-11 \mathrm{Am})$. All animal experiments were approved by the Animal Experimentation Ethics Committee of the Southern Medical University and were carried out in accordance with the guidelines set by the committee.

\subsection{The Spin-magnetic Field Generator}

The RMF was generated by a spin-magnetic field generator which was home-designed and made by Guangzhou Kenaifu dynamo Inc. It consists of four functional units: an aluminum plate, an $\mathrm{AC}$ motor, a velocity-control unit, and a velocity measurement unit. The aluminum plate is a round disk, made of pure aluminum with diameter $20 \mathrm{~cm}$ and thickness $14 \mathrm{~mm}$. There are twenty holes $(12 \mathrm{~mm}$ in diameter and

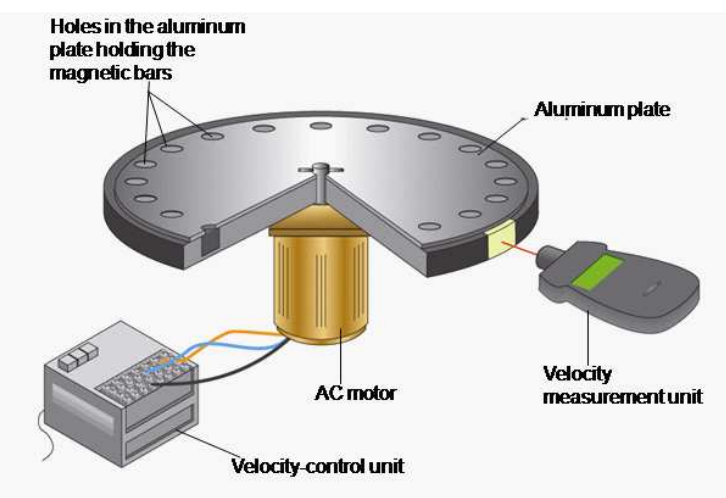

Figure 1. The spin-magnetic field generator, consisting of an aluminum plate with embedded magnetic bars, an AC motor, a velocity-control unit, and a velocity measurement unit. 
Table 1. The suppression of the paw withdraw reflex by rotating magnets.

\begin{tabular}{|c|c|c|c|c|}
\hline $\begin{array}{c}\text { Treatments } \\
\text { groups })\end{array}$ & $\begin{array}{c}\text { PWR }(-) \\
(n)\end{array}$ & $\begin{array}{c}\text { PWR }(+) \\
(n)\end{array}$ & $\begin{array}{c}\text { Total } \\
(n)\end{array}$ & $\begin{array}{c}\text { Suppression } \\
\text { rate }(\%)\end{array}$ \\
\hline $\begin{array}{c}\text { Spining } \\
\text { magnets }\end{array}$ & 11 & 1 & 12 & 91.67 \\
\hline $\begin{array}{c}\text { Spining alnico } \\
\text { alloy bars }\end{array}$ & 0 & 12 & 12 & 0 \\
\hline Total & 11 & 13 & 24 & 45.83 \\
\hline
\end{tabular}

$12 \mathrm{~mm}$ in depth) drilled in parallel to the perimeter of the plate, into each hole a cylinder shaped Al-Ni-Co permanent magnet is embedded. The magnetic flux density of these magnets is $0.68 \mathrm{~T}$, with the southpole on top. To securely anchor the magnets, the plate is covered by an aluminum slide ( $18 \mathrm{~cm}$ in diameter and $3 \mathrm{~mm}$ in thickness). Controlled by a frequency transformer, the AC motor (Hitachi, $90 \mathrm{~W}$ ) has a capacity of changing the angular velocity of magnets in the range of $0-15,000$ circles/min (Fig. 1).

\subsection{Paw Withdrawal Reflex}

The paw withdrawal reflex (PWR) was induced by clamping the toe of a rat with a constant force for a certain period of time. Briefly, the finger ring of a medium size hemostat was wrapped with a loop of rubber band (coefficient of elasticity is 12.75 Newton $/ \mathrm{m}$ ). When a clamping was performed, the rubber band was adjusted until the force generated by the hemostat was 1 Newton. Three clamping tests were performed for each animal and each test lasted for 1 second. A successful reflex was counted only when an animal responded to all three clamping.

\subsection{Tail Flick Test}

The tail flick (TF) test has been widely used to measure injury-related alterations in sensory threshold $[20,21]$. Briefly, a rat was placed in a plastic box $(22 \times 6.5 \times 6.5 \mathrm{~cm})$. The box contained two holes to allow the rat to protrude the head and tail, respectively. A heating stimulation $\left(40^{\circ} \mathrm{C}\right)$ was provided by a photo-thermal pain threshold detector $(\mathrm{ZH}-$ LUO/B, Zheng Hua Bio-instruments, Anhui, China). The latency (seconds) between a heat stimulation and a TF response was recorded. Based on our pilot experiments, a specific point between the middle and 
upper one third of the tail was stimulated in all rats to minimize the variability of the results, and the latency between the stimulation and a TF response was recorded. Animals with TF latency shorter than $5 \mathrm{~s}$ (hyper-sensitive) or longer than $10 \mathrm{~s}$ (hypo-sensitive) were excluded in the experiment. To minimize possible effects of stress on the TF response, rats were exposed to the rotating magnets at $6000 \mathrm{r} / \mathrm{min}$ for 10 minutes every day in the week before testing day. To minimize the risk of tissue damage, a 30 seconds cut-off was used as the maximal duration of heat stimulation.

\subsection{Mechanical Withdrawal Threshold}

The mechanical withdrawal threshold (MWT) was measured using a commercial device (Dynamic Plantar Aesthesiometer, Ugo Basile; Comerio, Italy). Prior to testing, rats were placed in the apparatus for at least 15 min or until they ceased exploratory behavior. The paw withdrawal was measured with a probe $(0.6 \mathrm{~mm}$ diameter $)$ placed close to the center of the plantar surface of the left hind paw for shorter than $4 \mathrm{~s}$. The force applied by the probe was ramped from 0.5 to $20 \mathrm{~g}$ over a $20 \mathrm{~s}$ period, and the minimal force that elicited a reflex removal of the paw was recorded.

\subsection{Statistical Analysis}

SPSS13.0 was used to analyze the data. All data were verified for normal distribution and homogeneity of variance. In-homogeneous data were modified with Welch's method. Data in the first experiment was analyzed by Chi-square test. One-way analysis of variance (ANOVA) was performed for the data in the experiments 2 and 3 , followed by $t$ tests as post-hoc comparisons. The student $t$ test was performed for experiments examining effects of spinning direction on pain threshold. Differences were considered statistically significant when $p<0.05$. In addition, the correlations between spinning velocity and TF latency/MWT and the correlations between the spinning time and TF latency/MWT were calculated.

\section{RESULTS}

\subsection{Rotating Magnets Suppress the Paw Withdraw Reflex (PWR) in Rats}

In the first experiment, 24 adult male rats weighing $200-250 \mathrm{~g}$ were used in two groups (12/group). After an anesthesia with $10 \%$ chloral hydrate $(300 \mathrm{mg} / \mathrm{kg})$, a rat was hung $5 \mathrm{~mm}$ above the magnetic plate. 
When the plate rotated (in the anti-clockwise), the lumbar region of the rat was passively cut by magnetic lines from the rotating magnets at a consistent angular velocity of 6000 circles/min (Fig. 2). Rats in the control group were subjected to the same experimental procedures, except that the magnets on the plate were replaced with the Alnico alloy bars of the same shape and size. The paw withdrawal reflex (PWR) of the anesthetized rats was measured immediately and 5 minutes after magnets stopped rotating, which continued for 10 minutes. After the anesthesia but before started rotating, all rats, in the absence (control group) or presence (experimental group) of the magnetic field, showed positive PWR, suggesting that the $10 \%$ chloral hydrate treatment and the static magnets did not block PWR in rats. Immediately after magnets rotating stopped, 11 of 12 rats in the experimental group showed no PWR, whereas all rats in the control group showed positive PWR (Table 1). Five minutes after the magnets stop rotating, the PWR re-appeared in all rats in the experimental group.

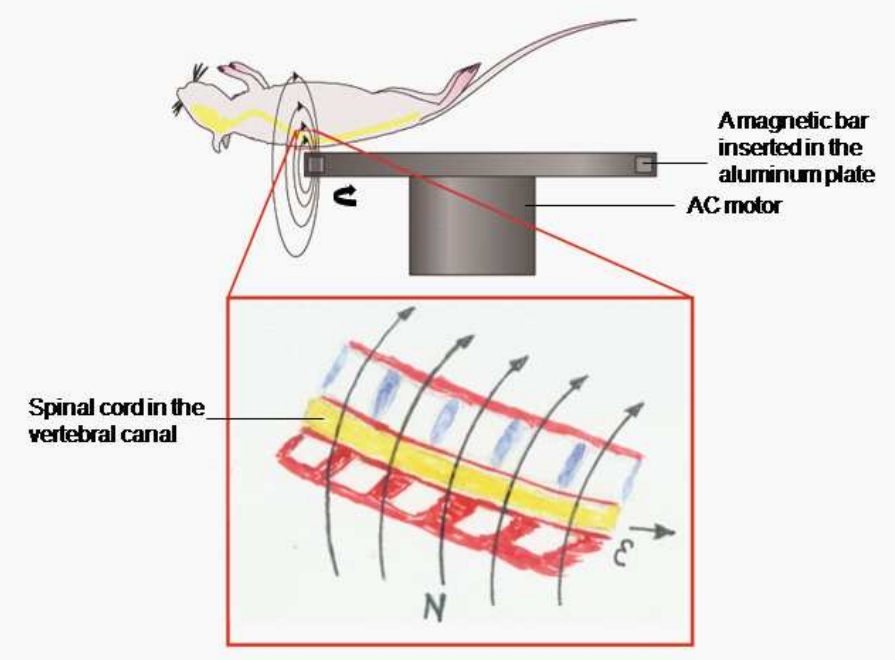

Figure 2. The position of a rat relative to the aluminum plate. When the plate rotates in clockwise or anti-clockwise directions (in the horizontal plane) the magnetic lines from the rotating magnetic bars penetrate through the lumbar region of the spine. The spinal cord in the spinal canal, the rotating direction of the aluminum plate, the direction of the magnetic lines $(N)$, and the direction of the induced current $(\varepsilon)$ were illustrated. 


\subsection{Rotating Magnets Increase Tail Flick Latency and Mechanical Withdrawal Threshold in Conscious Rats}

We next investigated if rotating magnets exert similar analgesia effect on conscious rats and if the treatment has the same effects on the other sensory-motor measurements. For this goal, the second experiment assessed TF latency and MWT. The TF test has been widely used to measure injury-related alterations in sensory threshold [20,21]. Forty adult female rats weighing $150-160 \mathrm{~g}$ were grouped evenly (10/group) and exposed to magnets rotating for 30 minutes at 6300,6800 , 7300 , or 7800 circles/min, respectively. Before and immediately after magnets stopped rotating, TF latency and MWT of conscious rats were measured. All rats that had been subjected to rotating magnets showed longer TF latency (Fig. 3(a)) and greater MWT (Fig. 3(b)) compared to the baselines. Moreover, the values of TF and MWT increased as the functions of the angular velocity of rotating. Welch modified one-way ANOVA indicated significant effects of the angular velocity on both the TF latency $(F(3,36)=127.81, P<0.001)$ and MWT $(F(3,36)=6.80, P<0.001)$. Post hoc comparisons revealed a significant increase in TF latency at all tested angular velocities. The largest increase occurred in rats subjected to the highest velocity ( 7800 circles $/ \mathrm{min})$ and the smallest but significant increase occurred in rats subjected to the relatively lower velocity (6300 circles/min). Similarly, MWT varied as the function of the angular velocity of magnets rotating.

The third experiment was done with 24 adult female rats weighing $150-160 \mathrm{~g}$ in four groups (6/group) to investigate effects of exposing time. Rats in the four groups were subjected to a same magnets rotating ( 7300 circles/min) for $10,20,30$, or 60 minutes, respectively. Their TF latency and MWT were measured before and immediately after magnets rotating stopped. A positive correlation between exposing time and TF was found (Fig. 4(a)). Welch modified one-way ANOVA indicated a significant effect of exposing time on TF latency $(F(3,20)=35.563, P<0.01)$. Post hoc comparisons revealed the most significant effect in the rats subjected to the magnets rotating for 60 minutes and a relatively smaller but significant effect in those exposed for 10 minutes. A similar positive correlation between exposing time and MWT was also found (Fig. 4(b)). These data provided further evidence for the analgesia effect of rotating magnets. 


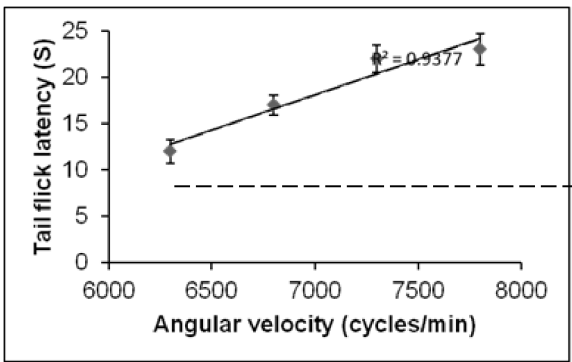

(a)

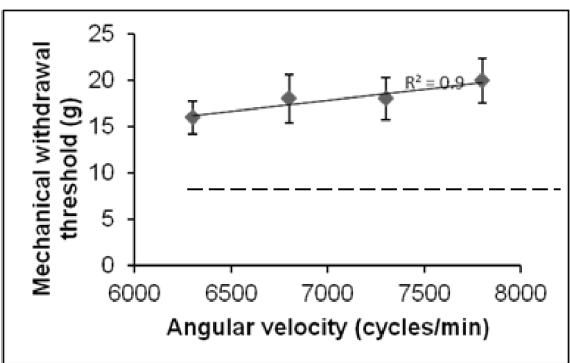

(b)

Figure 3. The correlations between the rotating speed of magnets and the TF latency/MWT of rats. (a) Conscious rats were exposed to static or rotating magnets for 30 minutes at the indicated angular velocities. The TF latency was measured before magnets rotating (static magnets, dash line) and immediately after rotating stopped. (b) Conscious rats were exposed to static or rotating magnets for 30 minutes at the indicated angular velocities. The MWT of them was measured before magnets rotating (static magnets, dash line) and immediately after rotating stopped.

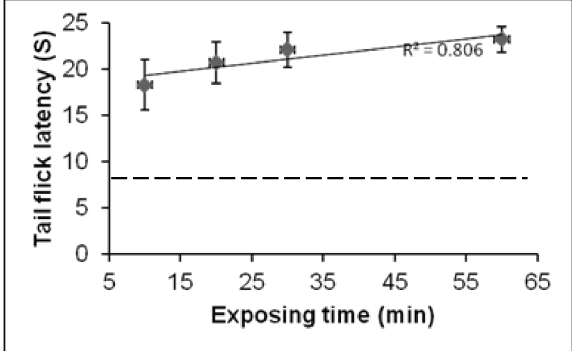

(a)

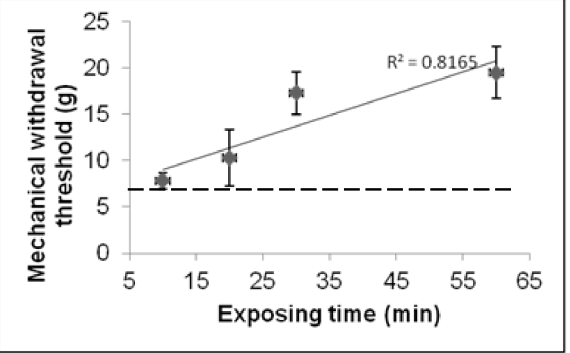

(b)

Figure 4. The correlations between the time exposing to rotating magnets and the TF latency/MWT. (a) Conscious rats were exposed to static or rotating magnets at 7300 circles $/ \mathrm{min}$ for the indicated periods. The TF latency of them was measured before magnets rotating (static magnets, dash line) and immediately after rotating stopped. (b) Conscious rats were exposed to static or rotating magnets at 7300 circles $/ \mathrm{min}$ for the indicated periods. The MWT of them was measured before magnets rotating (static magnets, dash line) and immediately after rotating stopped. 


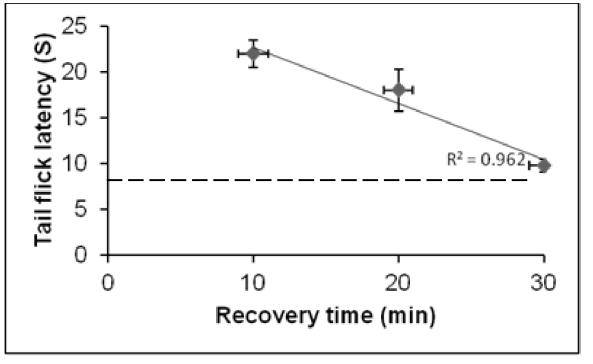

(a)

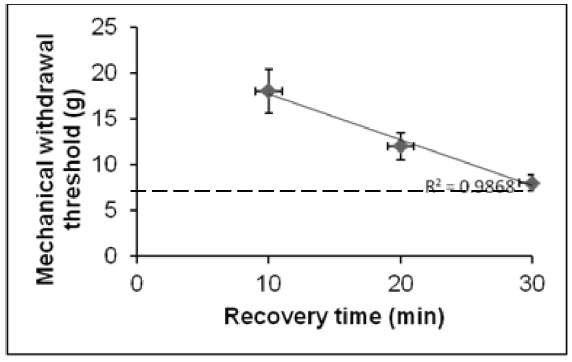

(b)

Figure 5. The recovery of rats from the rotating magnets induced analgesia effect. (a) Conscious rats were exposed to rotating magnets at 7300 circles $/ \mathrm{min}$ for 60 minutes. The TF latency was measured at the indicated time points. The $\mathrm{TF}$ value measured before rotating was used as the baseline (dash line). (b) Conscious rats were exposed to rotating magnets at 7300 circles/min for 60 minutes. The MWT was measured at the indicated time points. The MWT value measured before rotating was used as the baseline (dash line).

\subsection{Rats Rapidly Recover to Pre-exposing Levels from the Magnets Rotating-induced Analgesia Effects}

A corollary drawn from the positive correlations between the exposing time and TF latency/MWR is that recovering to the pre-exposing levels from the magnets rotating-induced analgesia would be a timedependent process. To confirm this corollary, a group of rats $(n=6)$ in the third experiment were subjected to a magnets rotating at 7300 circles/min for 60 minutes and their TF latency and MWR were measured 10, 20, or 30 minutes after magnets rotating stopped. As shown in Fig. 5(a), TF values of rats decreased when the recovery time increased and it (TF) recovered to the pre-exposing level 30 minutes after magnets rotating stopped. A similar time-dependent recovery process was revealed with the data of MWR (Fig. 5(b)).

\subsection{The Analgesia Effect is Independent of the Direction of the Rotation}

Magnet rotating in both directions induces the same analgesia effect. To investigate the effects of different field orientation relative to the rats, we exposed conscious rats to rotating magnets at 7300 circles $/ \mathrm{min}$ in both clockwise and anti-clock wise directions for 30 minutes. Both the TF latency and MWT were measured immediately after rotating stopped. We find that reversing the rotating direction yielded similar 


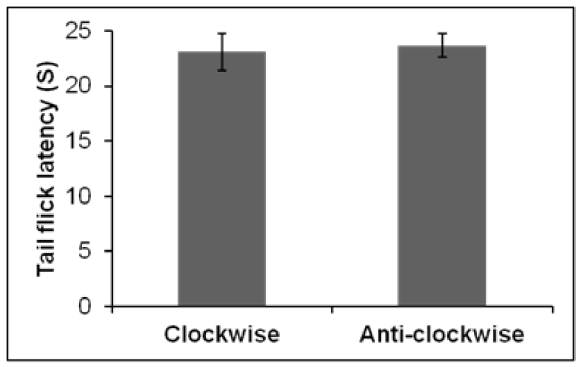

(a)

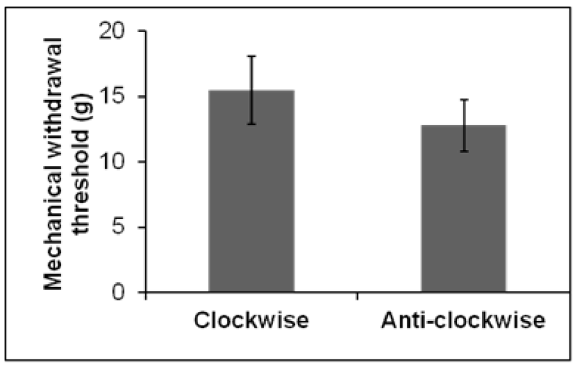

(b)

Figure 6. Magnet rotating in both directions induces the same analgesia effect. (a) Conscious rats were exposed to rotating magnets at 7300 circles/min in both clockwise and anti-clock wise directions for 30 minutes. The TF latency of them was measured immediately after rotating stopped. (b) Conscious rats were exposed to rotating magnets at 7300 circles/min in both clockwise and anti-clock wise directions for 30 minutes. The MWT of them was measured immediately after rotating stopped.

analgesia effect with identical TF latency and MWT (Fig. 6).

\section{DISCUSSION}

Our results demonstrated that rotating magnets produce analgesia effect in rats. This analgesia effect is likely due to the electric current induced by the time-varying RMF. The induced-current may depolarize or hyperpolarize the axonal membrane, thus blocks the conduction of action potential along axons. The electric field parallel to the axons has been shown to be the most effective in affecting the axonal membrane potential $[22,23]$. With this orientation, the axon membrane potential can be described by the modified cable equation $[22]$

$$
f=\lambda^{2} \frac{\partial^{2} V_{m}}{\partial x^{2}}-\tau \frac{\partial V_{m}}{\partial t}-V_{m}=-\lambda^{2} \frac{\partial V_{e}}{\partial x^{2}}
$$

where $\lambda^{2}=\frac{R_{m} a}{2 R_{i}}$ and $\tau=R_{m} C_{m}$ are the space and time constants, respectively. $V_{m}$ is the trans-membrane potential and $V_{e}$ is the extracellular electric field applied to the fiber. The surface resistance and capacitance of the membrane are $R_{m}$ and $C_{m}$, the intracellular resistivity is $R_{i}$ and the fiber radius is $a$. $f$ is called Rattay's activation function [24]. Negative value represents depolarization and positive one means hyper-polarization of cell membrane. 


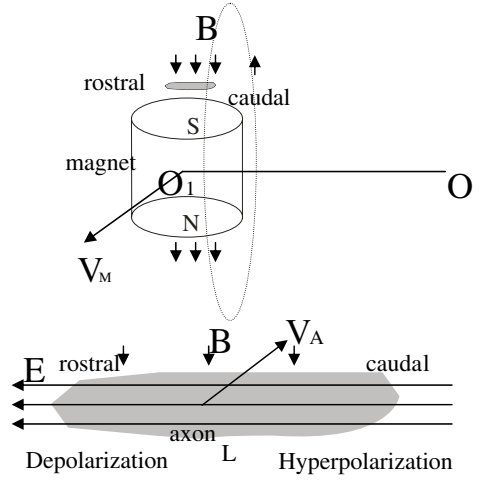

(a)

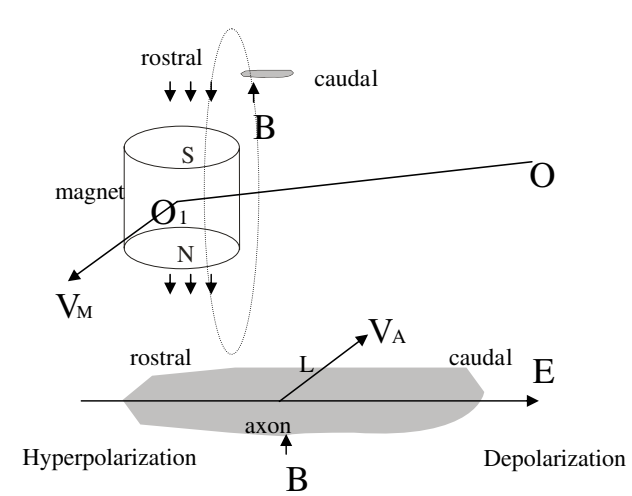

(b)

Figure 7. A biophysics model illustrating the depolarization/hyperpolarization of axons exposed to rotating magnets. (a) Orientation of a magnet and its movement, in relationship to a spinal axon segment when a rat is right on the top of the magnet. The cylinder represents a magnet centered at $O_{1}, O$ is the center of the spinning plate $\left(O O_{1}=20 \mathrm{~cm}\right) . \mathbf{V}_{\mathbf{M}}$ represents the movement of the magnet (corresponds to the counter-clockwise rotation of the whole plate). B is the magnetic field generated by the magnet $(S$ - South pole; $N$ - North pole). An axonal segment in the spinal cord was drawn above the magnet, with its rostral end on the left side, and caudal end on the right side. $\mathbf{L}$ is the length of the axon segment. $\mathbf{V}_{\mathbf{A}}$ represents the movement of the axon relative to the magnet during fast spinning. $\mathbf{E}$ is the electric field induced by the movement of the magnet, as determined by Flemings right hand rule. Magnetically induced electric current enters caudal end of the axon, generating membrane hyperpolzrization, and leaves the axon on its rostral end, generating membrane depolarization. (b) Polarization of the axon when the rat is not on top of the magnet. Compare to that shown in (a), the direction of the magnetic field has been reversed, and its density is much weaker. Electric current enters rostral end of the axon, generating membrane hyperpolarization, and leaves the axon on its caudal end, generating membrane depolarization.

The RMF induced-electric current may flow into or out of the axonal membrane, thus hyperpolarize or depolarize the membrane with the same outcome of blocking axonal conduction $[25,26]$. This explanation was well demonstrated in the study showing that a same magnets rotating (at 7300 circles $/ \mathrm{min}$ ) in both clockwise and counter-clockwise directions produced the same effects of prolonging 
TF latency and increasing MWT (Fig. 6). Based on the findings from this study and the connection between electricity and magnetism, a simple biophysics model was created (Fig. 7) to illustrate the orientation of a rotating magnet and its induced electric field with an assumed axon, and the locations where local de/hyper polarizations could occur on the axonal membrane. This model suggests that during fast-speed spinning, axonal segment experiences a dynamic local depolarization/hyperpolarization process. If the animal is right on top of the magnet, the axonal segment will be maximally polarized since the magnetic field intensity is at its maximum, and so is the induced electric field. The induced electric field will depolarize the rostral end of the axon, and hyperpolarize the caudal end (Fig. 7(a)). When the magnet moves away from the animal, direction of the induced electric field inside the axon is reversed and the axon experiences weaker local polarizations (Fig. 7(b)).

Based on this model, the induced electric potential by the magnetic field is in the longitudinal direction of the axon segment, and can be computed using the formula

$$
\varepsilon=V_{A} B L
$$

where $V_{A}=62.8 \mathrm{~m} / \mathrm{s}$ for a spin speed 100 cycle/second. $B(0.42 \mathrm{~T})$ is the intensity of the magnetic where the animal's spine was, above the magnets. Therefore, the maximal intensity of the induced electric field is about $E=26.4 \mathrm{~V} / \mathrm{m}$ along the axonal segment, as computed by $\varepsilon / L=V B$. Note the induced electric field is a function of the spinning speed, or the rotating frequency of the plate, this may explain the frequency-dependency of the pain threshold. This value is in a rational range with references to altered axonal states, exemplified by a recent study, in which a low intensity of electric field $(<10 \mathrm{~V} / \mathrm{m})$ was able to modulate the network dynamics in the hippocampus [27]. The same intensity of electric field was shown to suppress epileptiform activity in the hippocampal slices [28].

After magnets rotating stopped, it took 30 minutes for the increased TF latency and MWT to resume to pre-exposing levels (Fig. 5). To explain this phenomenon, some other factors need to be considered. First, the induced electric field may alter the potassium current involved in nerve conduction [29]. Second, the effect of static magnetic field $(0.68 \mathrm{~T})$ on the conduction of action potential along axons can't be completely excluded. For example, a $0.5 \mathrm{~T}$ magnetic field was able to block action potential conduction in the guinea pig spinal cord [2]. The magnetic field may also alter ion channel dynamics and the permeability of cell membrane to the charged ions, such as sodium and potassium, by applying Lorentz force. Although all these factors remain to be elucidated and warrant future studies, 
this study using a home-designed spin-magnetic field generator, for the first time, showed a prompt analgesia effect of induced electric current from rotating magnets. The induced electric current appeared to be non-invasive, while exerting analgesia effect. Further extensive studies inspired by this work will be eventually realizing a non-pharmacological approach of anesthesia.

\section{CONFLICT OF INTEREST STATEMENT}

The authors declare no competing financial interests.

\section{ACKNOWLEDGMENT}

This work is supported by National Natural Science Foundation of China (Grant No. 30870796/c090201), Science and Technology Planning of Guangdong Province (Grant No. 2009B030801223). We are grateful to Dr. Feng Changshen for his support and comments. The authors declare no competing financial interests.

\section{REFERENCES}

1. Cameron, T., "Safety and efficacy of spinal cord stimulation for the treatment of chronic pain: A 20-year literature review," Journal of Neurosurgery, 3 Suppl. Spine, Vol. 100, 254-67, 2004.

2. Coots, A., R. Shi, and A. D. Rosen, "Effect of a 0.5-T static magnetic field on conduction in guinea pig spinal cord," Journal of the Neurological Sciences, Vol. 222, Nos. 1-2, 55-7, 2004.

3. Kumar, K., et al., "Complications of spinal cord stimulation, suggestions to improve outcome, and financial impact," Journal of Neurosurgery, Spine, Vol. 5, No. 3, 191-203, 2006.

4. Rosenow, J. M., et al., "Failure modes of spinal cord stimulation hardware," Journal of Neurosurgery, Spine, Vol. 5, No. 3, 183-190, 2006.

5. De Leon-Casasola, O. A., "Spinal cord and peripheral nerve stimulation techniques for neuropathic pain," Journal of Pain and Symptom Management, 2 Suppl., Vol. 38, 28-38, 2009.

6. Kovacs-Balint, Z., et al., "Exposure to an inhomogeneous static magnetic field increases thermal pain threshold in healthy human volunteers," Bioelectromagnetics, Vol. 32, No. 2, 131-139, 2011.

7. Laszlo, J. and N. Pivec, "Effect of inhomogeneous static magnetic field on dental pain in humans," The Clinical Journal of Pain, Vol. 26, No. 1, 49-55, 2010. 
8. Thomas, A. W., et al., "A randomized, double-blind, placebocontrolled clinical trial using a low-frequency magnetic field in the treatment of musculoskeletal chronic pain," Pain Research \& Management: The Journal of the Canadian Pain Society, Vol. 12, No. 4, 249-258, 2007.

9. Eccles, N. K., "A critical review of randomized controlled trials of static magnets for pain relief," Journal of Alternative and Complementary Medicine, Vol. 11, No. 3, 495-509, 2005.

10. Hong, C. Z., et al., "Magnetic necklace: Its therapeutic effectiveness on neck and shoulder pain," Archives of Physical Medicine and Rehabilitation, Vol. 63, No. 10, 462-466, 1982.

11. Salomonowitz, G., M. Friedrich, and B. J. Guntert, "Medical relevance of magnetic fields in pain therapy," Schmerz, Vol. 25, No. 2, 157-160, 162-165, 2011.

12. Khoromi, S., et al., "Low intensity permanent magnets in the treatment of chronic lumbar radicular pain," Journal of Pain and Symptom Management, Vol. 34, No. 4, 434-445, 2007.

13. Thuile, C. and M. Walzl, "Evaluation of electromagnetic fields in the treatment of pain in patients with lumbar radiculopathy or the whiplash syndrome," NeuroRehabilitation, Vol. 17, No. 1, 63-67, 2002.

14. Saunders, R., "Static magnetic fields: Animal studies," Progress in Biophysics and Molecular Biology, Vol. 87, Nos. 2-3, 225-239, 2005.

15. Wikswo, J. P. and J. P. Barach, "An estimate of the steady magnetic field strength required to influence nerve conduction," IEEE Transactions on Bio-medical Engineering, Vol. 27, No. 12, 722-723, 1980.

16. Lefaucheur, J. P., X. Drouot, and J. P. Nguyen, "Interventional neurophysiology for pain control: Duration of pain relief following repetitive transcranial magnetic stimulation of the motor cortex," Neurophysiologie Clinique, Vol. 31, No. 4, 247-252, 2001.

17. Sampson, S. M., et al., "The use of slow-frequency prefrontal repetitive transcranial magnetic stimulation in refractory neuropathic pain," The Journal of ECT, Vol. 27, No. 1, 33-37, 2011.

18. Vavken, P., et al., "Effectiveness of pulsed electromagnetic field therapy in the management of osteoarthritis of the knee: A metaanalysis of randomized controlled trials," Journal of Rehabilitation Medicine: Official Journal of the UEMS European Board of Physical and Rehabilitation Medicine, Vol. 41, No. 6, 406-411, 2009. 
19. Shupak, N. M., et al., "Exposure to a specific pulsed low-frequency magnetic field: A double-blind placebo-controlled study of effects on pain ratings in rheumatoid arthritis and fibromyalgia patients," Pain Research \& Management: The Journal of the Canadian Pain Society, Vol. 11, No. 2, 85-90, 2006.

20. Kaneko, M., et al., "Synergistic antinociceptive interaction after epidural coadministration of morphine and lidocaine in rats," Anesthesiology, Vol. 80, No. 1, 137-150, 1994.

21. Yamamori, Y., et al., "Antinociceptive effects of ONO-9902, an enkephalinase inhibitor, after visceral stress condition in rats," Canadian Journal of Anaesthesia, Vol. 43, No. 11, 1175-1179, 1996.

22. Roth, B. J. and P. J. Basser, "A model of the stimulation of a nerve fiber by electromagnetic induction," IEEE Transactions on Bio-medical Engineering, Vol. 37, No. 6, 588-597, 1990.

23. Ye, H., et al., "Transmembrane potential generated by a magnetically induced transverse electric field in a cylindrical axonal model," Medical \& Biological Engineering \& Computing, Vol. 49, No. 1, 107-119, 2011.

24. Rattay, F., "Analysis of models for external stimulation of axons," IEEE Transactions on Bio-medical Engineering, Vol. 33, No. 10, 974-977, 1986.

25. Bhadra, N., et al., "Simulation of high-frequency sinusoidal electrical block of mammalian myelinated axons," Journal of Computational Neuroscience, Vol. 22, No. 3, 313-326, 2007.

26. Kilgore, K. L. and N. Bhadra, "High frequency mammalian nerve conduction block: Simulations and experiments," Conference Proceedings: Annual International Conference of the IEEE Engineering in Medicine and Biology Society, Vol. 1, 4971-4974, 2006.

27. Reato, D., et al., "Low-intensity electrical stimulation affects network dynamics by modulating population rate and spike timing," The Journal of Neuroscience: The official journal of the Society for Neuroscience, Vol. 30, No. 45, 15067-15079, 2010.

28. Gluckman, B. J., et al., "Electric field suppression of epileptiform activity in hippocampal slices," Journal of Neurophysiology, Vol. 76, No. 6, 4202-4205, 1996.

29. Liu, H., et al., "The role of slow potassium current in nerve conduction block induced by high-frequency biphasic electrical current," IEEE Transactions on Bio-medical Engineering, Vol. 56, No. 1, 137-46, 2009. 